\title{
Perceptions, Facilitators And Barriers of Physical Activity Among People Living With HIV: A Qualitative Study
}

Brenda Kitilya ( $\sim$ brendawilfredkitilya@yahoo.co.uk)

National Institute for Medical Research

Erica Sanga

National Institute for Medical Research

George PrayGod

National Institute for Medical Research

Robert Peck

Weill Bugando School of Medicine

\section{Bazil B Kavishe}

National Institute for Medical Research

Kia Ditlevsen

University of Copenhagen

Mette F Olsen

University of Copenhagen

\section{Research Article}

Keywords: People Living with HIV, Perceptions, Facilitators, Barriers, Physical activity

Posted Date: June 24th, 2021

DOl: https://doi.org/10.21203/rs.3.rs-567121/v1

License: (c) (i) This work is licensed under a Creative Commons Attribution 4.0 International License. Read Full License 


\section{Abstract}

\section{Background}

People Living with HIV (PLWH) have low levels of physical activity. Understanding of perceptions, facilitators and barriers of physical activity in this population is of importance for future perspectives in developing contextual based intervention to improve physical activity in PLWH.

\section{Method}

This study explored perceptions, facilitators and barriers of physical activity in PLWH using sixteen indepth interviews and three focus group discussions conducted in Mwanza, Tanzania. The interviews and discussions were audio recorded, transcribed and translated into English. Transcripts were coded and analysed deductively using thematic content analysis. The themes were interpreted and presented using the social ecological model.

\section{Results}

Forty-three PLWH age 23-61 years old participated in this study. The findings showed that, PLWH perceived physical activity was beneficial to their health and that their perceptions and practices were rooted within the existing gender stereotypes and roles in the community. In addition to that, housechores and income generating activities were perceived as sufficient physical activity to achieve adequate levels of physical activity. As facilitators, social support and interactions from family and friends were appraised positively as motivating factors of physical activity in this population. Indeed, at individual to community/environmental levels, PLWH reported barriers of physical activity were lack of time, money, and availability of facilities, as well as lack of social groups participating in physical activity in the communities and inadequate information on physical activity from health care providers at ART clinics. HIV disease was not reported as a main barrier of physical activity but rather stipulated from family members as a reason not to support physical activity in PLWH.

\section{Conclusion}

The findings demonstrated differing perceptions, facilitators and barriers of physical activity among PLWH. Interventions addressing physical activity awareness, gender stereotypes and roles relating to physical activity performances from individual to community level, building supportive environment and infrastructures are needed to elevate physical activity levels in PLWH particularly in low and -middle income countries.

\section{Background}

Physical activity in People Living with HIV (PLWH) may ameliorate the side effects of antiretroviral therapy (ART) and improve quality of life overall [1-3]. Despite these health benefits, studies have shown that PLWH have low levels of physical activity [4]. Besides biological drivers of reduced physical activity, 
such as chronic inflammation, ART side effects and poor nutrition [5], studies have reported a number of behavioural and socio-cultural attributes which contributes to low level of physical activity. These includes lack of time, knowledge, motivation, physical appearance concerns, tiredness from work, lack of resources to pay for using physical activity facilities and lack of support from the family and community members, demographic factors and low income [6-8].

The majority of studies assessing underlying factors contributing to low physical activity in adults, including PLWH have been conducted in high-income countries [9]. Studies included evaluations on participation and motivation at individual or community-based physical activity programs [2, 10-12]. However, in Sub-Saharan Africa (SSA), studies on HIV and physical activity are scarce [13].

Previous studies have used several theoretical models of behavioural change including the health belief model, transtheoretical model and the social-cognitive model to describe factors, motivation and adherence to physical activity at individual level [14-16]. Critics on these health-behavioural models have argued that, changes focusing on individual's lifestyle or behaviour including physical activity needs more than psychological-behaviour approach, since individuals make decisions or choices within a social-ecological context [17] and that includes the social ecological model (SEM) approach with intrapersonal, interpersonal, community/environment and policy influences [18]. These critics have called for qualitative studies to provide in-depth understanding of contextual influences of behaviour, including physical activity [19] and studies have used SEM to create and maintain interventions or programs which facilitates communities to actively engage in physical activity $[18,20,21]$. Thus, we aimed to explore the perceptions, facilitators and barriers of physical activity among PLWH by utilizing the socio-ecological model (SEM).

\section{Method}

\section{Study setting and design}

This was a descriptive qualitative study conducted in Mwanza Region in the North -Western part of Tanzania in the month August-November, 2019. The study was nested within a cohort study, "Chronic Infection, Co-morbidities And Diabetes in Africa (CICADA)" registered at http://clinicaltrials.gov as NCT03106480. Details of the CICADA study design has been published [22]. Mwanza has a population of $\sim 2.7$ million and a rate of HIV-prevalence of 9.2\% [23]. The common ethnic groups found in the region are Sukuma, Haya, Jita and Kerewe and the main income generating activities performed in this region are petty trading, fishing, livestock keeping, farming and mining [23].

\section{Social ecological model}

This study deployed SEM adopted from McElroy 1988 [18], to understand in-depth the underlying perceptions, facilitators and barriers of physical activity in PLWH Fig. 1. The SEM has intrapersonal, interpersonal, organizational, community and public policy levels. However, for this study, organizational and public policy level were incorporated at the community/environmental level because of overlapping 
of the influences and the occurrence of the multifaceted factors in those levels are at large in the communities such as implementation of public policies and public institutions supporting physical activity [24].

\section{Participants}

Purposive sampling was used to recruit 43 adult participants from the CICADA cohort with representation from different sexes, age groups, occupations and marital status. All of the recruited participants had HIV for more than 1 year and were on ART services Table 1.

Table 1

Characteristics of People Living with HIV participating in In-depth interview and Focus group discussions

\begin{tabular}{|ll|}
\hline Characteristics & Number of participants (n/\%) \\
\hline Gender & $23(53.5 \%)$ \\
\hline Female & $20(46.5 \%)$ \\
\hline Male & \\
\hline Age group (years) & $3(7.0 \%)$ \\
\hline $18-25$ & $10(23.2 \%$ \\
\hline $16-30$ & $24(55.8 \%)$ \\
\hline $31-45$ & $6(14 \%)$ \\
\hline $46-65$ & $33(76.7 \%)$ \\
\hline Time since diagnosis of HIV (years) & $10(23.3 \%)$ \\
\hline $1-3$ & \\
\hline$>3$ & $9(20.9 \%)$ \\
\hline Marital status & $10(23.3 \%)$ \\
\hline Single & $18(41.9 \%)$ \\
\hline Married & $6(14.0 \%)$ \\
\hline Divorce/ Separated & $30(69.8 \%)$ \\
\hline Widow/widower & $7(16.3 \%)$ \\
\hline Occupation & \\
\hline Business owner/Petty trader & \\
\hline Employed & \\
\hline Unemployed/housewives & \\
\hline
\end{tabular}




\section{Data collection, management and analysis procedures}

Data collection, management and analysis were conducted concurrently in an iterative process. Sixteen In-Depth Interviews (IDIs) were used to capture individual detailed information on perceptions, facilitators and barriers of physical activity whereas three Focus-Group Discussions (FGD's) were used to capture social-cultural norms on perceptions and barriers of physical activity in the communities [25]. The number of IDIs and FGDs was determined by when a data saturation point was reached.

The topic guides for the IDIs and FGDs were developed in English based on the theoretical perspectives of SEM and applied to physical activity [26]. Open-ended questions on perceptions, facilitators and barriers reflecting individual, social and family to community factors influencing physical activity were included in the topic guides. The topic guides were translated to Kiswahili language, pilot-tested in separate interviews to assess language, concept and content meaning of the questions and were revised accordingly before they were used in the study. Data was collected by the first and second authors (BK and ES) who were both familiar with the studied population. The interviews and group discussions were conducted by starting to build a rapport and comfortability of the participants in order to improve their understanding of the questions and responses in the discussion process. For FGDs, the first group was for men, second group was for women and third group included both. BK and ES worked together interchangeably as an interviewer or a note taker during the data collection process. The IDIs lasted 30 minutes while FGDs lasted between 30-45 minutes.

IDIs and FGDs were audio recorded using Sony tape recorder (IC recorder, ICD PX470, China) and fully transcribed and translated to English by a research assistant independent of the researchers who collected the data. For quality control, a sample of the transcripts were compared with the audio recordings by BK and ES.

Data analysis was conducted independently by BK and ES. Where there were any disagreements on the data, the two researchers resolved by discussions until consensus was reached. Analytical deductive content analysis was conducted to identify and extract findings in a procedure described by Elos and Kyngas [27]. The procedures for analysis were as follows: firstly, the transcripts were thoroughly read and categorised resulting in the following units of analysis (major themes): 1) perceptions of physical activity, 2) facilitators of physical activity and 3) barriers of physical activity. Each unit of analysis was analysed and interpreted in three dimensions: the individual, social and family, and community/environmental level. In particular, the analysis of perceptions, facilitators and barriers of physical activity at individual, social and family level were based on data from IDIs. Analysis of community perceptions, facilitators, and barriers of physical activity were based on data from FGDs. Secondly, the researcher extracted the text notes from the transcriptions and categorised them into themes relating to perceptions, facilitators and barriers of physical activity at the individual, social and family, and community/environmental level. The categorisation of themes was based on content characteristics. For example, for perceptions at individual level, themes were knowledge and interpretations of physical activity, importance of physical 
activity, and practices in terms of types and amount of physical activity. Thirdly, the themes were interpreted and presented with participants quotes as illustrations of what was reported.

\section{Ethical considerations}

Ethical approval for the study was provided by the Medical Research Coordinating Committee of the National Institute for Medical Research and Catholic University of Health and Allied Sciences Ethics Review Board. All eligible participants were informed of the study purpose and procedures including voluntary participation, right to withdraw at anytime from the study procedures without any consequences attached to their action. Participants provided a written informed consent prior to their enrolment to a member of the research team. Confidentiality and anonymity was maintained throughout the study and numbers were used to represent participants.

\section{Results}

Findings are presented in three major themes: 1) perceptions of physical activity, 2) facilitators of physical activity and 3) barriers of physical activity. All themes are described at the individual, social and family, and community/environmental level.

\section{Perceptions of physical activity among PLWH at the individual level}

Participants revealed differing interpretation of the terms "physical activity" and "exercise". The term "physical activity" was translated to ("Shughuli za mwill" in Swahili) which was perceived as an abstract concept, while the "exercise" ("mazoezi" in Swahili) was a more commonly used term. Participants would generally describe physical activity practices with examples using the word "exercise". On occasions, participant's distinct physical activity with certain practices including house chores and income generating activities involving walking long distances were described as "physical activity", in contrast to "exercise" which was perceived as recreational activities with intentions of improving physical strength or endurance. Participants also perceived that income generating activities such as petty trading, walking long distances selling fruits were sufficient physical activity. In addition, the commonly mentioned physical activity practices were walking, running, rope skipping and playing football of which were reported to be easy to perform and suitable for many age-groups. For example, as reported by a participant:

"Physical activities can mean work that you can do at home maybe to clean a house by mopping the floor or cultivating surrounding grasses and exercise means maybe walking, rope skipping, going to the gym" [IDI-32 year old women].

Some participants perceived sexual intercourse was part of physical activity and this was reported at individual level as well as from participants discussions reflecting community perceptions. For example, as explained by a participant: 
"Physical ... sexual intercourse with a woman is an exercise" [IDI-47 year old man].

Further, participants working in small scale businesses, walking long distances perceived that the amount of walking and work they do is sufficient physical activity.

Based on these quotations, inadequate social understanding of physical activity which is subjected to the commonly used words representing physical activity created confusion and discouraged the efforts of PLWH need to put on physical activity performances.

\section{Perceptions of physical activity among PLWH at the community level}

Apart from the individual perceptions of physical activity in participants, community social -cultural shared values can also contribute to individual choices or behavior of physical activity. In the group discussions, participants expressed perceptions of physical activity practices which were rooted within the existing gender stereotypes and roles in the community. Thus, men were seen as being more active than women and out-door recreational activities were seen as appropriate for men while house-chores were for women. As mentioned in a discussion:

"(In) the society that surrounds me, men do the exercise like running though it's just few of them. Women are only doing physical activities like house-chores and take it as exercise" [FGD-34 year old woman].

In addition, participants reported that other community members would associate certain types of exercise such as weightlifting, body building exercises practiced in groups, with criminal acts in preparation for robbery, burglary or other crime. This was reported in two FGDs and supported by the participants. As reported by participants in a group discussion:

"I remember I started exercising with my friend. We organized ourselves and then we started to exercise. After a short time, we inspired some other people and then formulated a group of seven people. After that we found a place which was an unfinished house. Then we created local equipment for our gym, but some people in the community don't know the meaning of exercise. They think that exercise is a source of crime and terrorism, so they reported us to the street leaders. Eventually, (the street leader) came at our gym. He saw our place then took our gym equipment. He took that equipment with him thinking that exercise is the source of terrorism and crime in the community. After a few days he came back again. He realised we were just doing exercise nothing else" [FGD-27 year old man].

PLWH women showed lack of enough confidence and competence to participate in out-door physical activity because of the underlying social-cultural rooted stereotypes and gender roles. In addition, poor interpretation of physical activity in the community, has led to contradicting efforts of encouragements and discouragements for PLWH willing to do physical activity.

\section{Facilitators of physical activity among PLWH at the individual, social and family levels}


Participants reported that the health benefits of physical activity, social support and interactions related to physical activity from family members and friends were the main facilitators of physical activity.

Participants felt motivated to do physical activity because it restores their physical abilities and improves their health. Self-efficacy and self-esteem would also improve due to the ability to appear healthy and not be immediately recognized as PLWH. As explained by the participant:

"What inspires me is, I want my body to be healthy and fit even if I am sitting among the people living with HIV, I cannot to be recognized (as HIV-infected). I do it for my health, I want to stay well" IIDI-32 year old woman].

Family and friends' encouragements and participation in physical activity practices was paramount in motivating participants in physical activity. As reported by a participant in an interview:

"We play with the children in my area, like rope jumping and sometimes we play netball... I even sometimes play football, I play with the youths there in our street" [IDI-32 years old woman].

\section{Facilitators of physical activity among PLWH at the community level}

The presence and access to football pitches and open spaces in the community for physical activity was mentioned as one of the facilitators of physical activity. As reported by the participants:

"In community there is no gym, but we have football pitches and many people use it to run" [FGD-45 year old men].

\section{Barriers of physical activity among PLWH at the individual, social and family level}

Participants commented that the main barrier of physical activity was time. Participants did not have enough time to do physical activity because of work-related activities. As a participant explained in an interview:

"I wish to walk more distance... I don't have enough time... sometimes you find that I have a lot of work and there is no time to go walking" [IDI 23 years old woman].

Some participants also feared physical activity would worsen their HIV disease leading to more deterioration of their heath rather than development of physical strength. As it was reported by a participant:

"After diagnosis, I thought maybe doing hard work will make me weak, I thought like I will die and leave my children, but after some time I was okay" [IDI-45 years woman].

Similarly, participants also reported that family members perceived physical activity practices to potentially worsen their HIV disease and for that matter, family members discouraged them to do 
physical activity. However, this was mentioned by few participants. As reported by the participant:

"My mother she doesn't advices me" she fears my condition [IDI-30 years old man].

Further, some family members discouraged physical activity due to lack of their time to support in these physical activities. This was observed, that even the immediate family members did not discuss or advise any issues pertaining physical activity for participants. As reported by the participant:

"Neither her husband nor her children tell her about doing any exercises" [IDI-36 years old woman].

Lack of resources or money to pay for access to fitness centers was also reported as a barrier of physical activity. As highlighted by the participant:

"I won't agree if they tell me to pay ten thousand (for gym) while I earn five hundred thousands, it will be difficult ... my income has to be in line with the gym costs" [IDI-61 years old man].

\section{Barriers of physical activity among PLWH at the community level}

Lack of social groups participating in physical activity was also reported as a barrier to physical activity in participants. In addition, the lack of promotional physical activity messages from health officials and other government officials was also perceived as a barrier. The health care professionals mainly emphasize on maintaining a healthy diet, good hygiene and ART treatment adherence. As reported by a participant in a group discussion:

"There is no place or groups for doing exercises. They never talk about exercise for sure" [FGD-43 years old man].

PLWH have clearly revealed that the main barriers to physical activity in the community were poor social support and lack of conducive environment to do physical activity. Healthcare professionals often do not deliver good advice to PLWH on physical activity.

\section{Discussion}

Our findings showed differing perceptions, facilitators and barriers to physical activity among PLWH from the individual, social and family levels through to the community/environmental level. This highlights the major influences both positive and negative that impact choices and decisions in performing physical activity in PLWH. PLWH revealed their own conceptualization of physical activity from individual, social and family to community level.

PLWH referred to physical activity as house-hold activities, income generating activities and regarded the day-to-day activities as a sufficient level of physical activity while exercise was regarded as recreational or geared towards building physical strength. However, the majority perceived that physical activity improves their physical strength, self efficacy and health in general. Such perceptions of the type, amount 
and practices of physical activity informs us about the inadequate understanding of physical activity which may contribute to low levels of physical activity in PLWH. Lack of knowledge and misperceptions on the guidelines pertaining to physical activity inhibits physical activity and may underestimate or overestimate the amount of exercise an individual performs $[28,29]$. A study that was conducted in China highlighted similar findings indicating that the level of participation in physical activity depends on the knowledge and understanding the benefits of physical activity [30]. This study clearly indicates a lack of awareness and misinterpretation of physical activity in PLWH. Initiations of physical activity programs with the goal of raising awareness and general knowledge of physical activity in PLWH may lead to improvement of existing local guidelines and in turn support physical activity in this population.

Existing gender stereotypes and gender roles influences physical activity participation and choices across cultures and communities [31]. The present study also found that PLWH reported gender differences in physical activity. Certain physical activities were perceived as either for men or women such as football for men and household-chores for women. Also, it was perceived that men do more physical activity than women. Similar findings were reported in a study conducted in Canada on gender stereotypes and physical activity. The study highlighted certain physical activities were perceived predominantly for boys and "less cool" for girls and boys were more active in physical activity while girls spend more time in socialisation [31]. Therefore, although this study reports gender stereotypes in the young population, it argues that participation in physical activity and range of sports at an earlier age contributes to a more flexible attitude later on toward gender/norms in physical activity [32]. In addition, a systematic review conducted in SSA reported that the traditional gender stereotypes of women not engaging in out-door activities influenced the level of physical activity participation because physical activity was perceived as unfeminine [33]. Findings from this study emphasize the need for contextual gender-based interventions appropriate for PLWH as well as interventions addressing positively the existing stereotypes and roles to accommodate and promote physical activity to improve health.

In this study, we also observed social support and interactions from family members and friends were facilitators of physical activity in PLWH. This social support in physical activity has been widely studied and has been noted to be a strong facilitator of physical activity. Other studies have gone further by indicating clearly the directions and differences of social support from friends and family members that could influence the motivations and maintenance of physical activity practices [34-36]. Furthermore, PLWH reported benefits resulting from doing physical activity such as increased physical strength, selfefficacy and improved health motivated them to initiate and maintain physical activity [12, 37]. However, it has also been argued that the concerns of PLWH for health improvements and improved physical appearance is among the fundamental coping strategies to reduce HIV related stigma [21]. Overall, our findings provide evidence for the value of social support to PLWH in physical activity. Interventions directly emphasizing on improving social and moral support to PLWH will aid promoting physical activity in this vulnerable population.

Barriers to physical activity reported among PLWH were time availability, lack of facilities and costs for recreational activities at fitness centers. In addition, they reported lack of encouragement from friends 
and family members, and health concerns due to HIV infection. Similar results were reported in other studies although most were conducted in older, women with HIV in high income countries [38, 39]. Other studies merely used SEM and a comparison group of HIV-uninfected individuals to explore barriers of physical activity [39]. These studies described biological factors such as physical exertion, opportunistic infections, presence of bodily pain, and depression as well as social and cultural factors including family discouragements, time constraints and unfamiliarity with physical activity facilities and machines in fitness centers as significant barriers to physical activity [7,39-41]. On the contrary, cultural issues relating to religion, cultural identity, body image and physical appearance were not identified as barriers to physical activity in our study, unlike in other studies $[38,41]$. Findings observed in this study on barriers may inform development of strategic and appropriately based interventions for PLWH to improve their physical activity practices in this context and reduce long-term effects of NCDs.

Apart from social barriers, lack of detailed information and encouragement on physical activity from health care services is among the barriers in the promotion of physical activity. This study reported poor sharing of information on physical activity at the ART clinics from health care professionals. Although the Tanzanian government's management and care of HIV and AIDS guideline has clearly stipulated that in middle and later stages of HIV infection, physical activity should be recommended to patients [42], this was not clearly portrayed by PLWH in our research. On the other hand, in the guideline, the recommendations for physical activity are not detailed and are merely mentioned as a sub-topic in relation to diet [42]. Therefore, in practice it can easily be overlooked or not stressed enough by health professionals. Nevertheless, the Tanzanian guideline have been criticised due its lack of alignment with the local concerns for ART service delivery [43]. Furthermore, even though the guideline has been updated over the years, the recent guideline pertaining to physical activity recommendations is incomplete and lacks detailed information [44]. Clinical practice guidelines need to clearly describe the terms and conditions of managing a certain disease in the context of available health systems [43], since physical activity is important in maintaining the health status of PLWH accessing ART services [45]. The findings of our study adds new information on how to incorporate physical activity promotional messages in the management of HIV and can inform guidelines in the ART clinics to improve advice on physical activity to PLWH.

\section{The Strength And Limitations Of The Study}

The strengths of this study include the use of SEM to explore in-depth the concepts and the context of physical activity and the social and cultural factors influencing physical activity in PLWH. In addition, to our knowledge this is the first study to explore social and cultural factors affecting physical activity in PLWH in Tanzania, SSA.

One of the limitations of this study is that it has been conducted in only one city in Tanzania and thus, the results may not be generalized to all settings. Secondly, the selection bias was unavoidable, that is to say the participants who were interviewed, were involved because they had time and enthusiasm to participate. 


\section{Conclusions}

The current study found there's a differing interpretation of physical activity and physical activity practices are rooted within existing gender stereotypes/roles in the communities. Further, PLWH felt motivated to do physical activity because of the encouragements from family members and friends and outcomes related from doing physical activity which were physical strength and improved health. However, reported barriers to physical activity were lack of time, limited awareness, health concerns related to HIV-infection, lack of support from family and friends, resources to pay for access to fitness centers, lack of social groups for physical activity and facilities. Further, the existing gaps in implementing the management and care of PLWH guidelines in relation to physical activity was also observed as a barrier to physical activity in PLWH. These results will support development of new strategic interventions in undertaking and focusing on the understanding of physical activity emphasizing on family and friends support in physical activity, wide dissemination of promotional messages in healthcare services and building supportive environments including facilities for physical activity in the communities to improve their health and well being of PLWH.

\section{Declarations}

Ethical approval and consent to participate: This study was conducted in accordance with the Declaration of Helsinki of 1964. Ethical approval for the study was provided by the Medical Research Coordinating Committee of the National Institute for Medical Research and Catholic University of Health and Allied Sciences Ethics Review Board. Participants were informed of the study purpose and procedures and provided a written informed consent prior to their enrolment.

\section{Consent for publication: N/A}

Availability of data: The datasets use/or analysed during the current study are available from the corresponding author on reasonable request.

Competing interests: the authors declare no conflict of interest.

Funding: This study was funded by the Ministry of Foreign Affairs of Denmark and administered by Danida Fellowship Centre (grant: 16-P01-TAN). The funding agency had no role in the study design, data collection and analysis, decision to publish results or preparation of the manuscript.

Authors' contributions: BK participated in the design, data collection, analysis, drafting and revised the final manuscript. ES was involved in the data collection, analysis, reviews and approved the final manuscript. GP, RP, BBK, and KD involved in the review process and approved the final manuscript. MFO participated in the design, review process and approved the final manuscript.

Acknowledgement: The authors thank all participants for participating in this study. We are grateful to the staff of the CICADA clinic, ART clinics in Mwanza. The Director General of NIMR and chairman of MRCC 
and Catholic University of Health and Allied Sciences is thanked for giving permission to publish this paper.

\section{References}

1. Rehm KE, Konkle-Parker D: Physical activity levels and perceived benefits and barriers to physical activity in HIV-infected women living in the deep south of the United States. AIDS care 2016, 28(9):1205-1210.

2. Botros D, Somarriba G, Neri D, Miller TL: Interventions to address chronic disease and HIV: strategies to promote exercise and nutrition among HIV-infected individuals. Current HIV/AIDS reports 2012, 9(4):351-363.

3. Nixon S, O'Brien K, Glazier RH, Tynan AM: Aerobic exercise interventions for adults living with HIV/AIDS. The Cochrane database of systematic reviews 2005(2):CD001796.

4. Schuelter-Trevisol F, Wolff FH, Alencastro PR, Grigoletti S, Ikeda ML, Brandao AB, Barcellos NT, Fuchs SC: Physical activity: do patients infected with HIV practice? How much? A systematic review. Current HIV research 2012, 10(6):487-497.

5. Olsen MF, Kaestel P, Tesfaye M, Abdissa A, Yilma D, Girma T, Molgaard C, Faurholt-Jepsen D, Christensen DL, Brage $S$ et al: Physical activity and capacity at initiation of antiretroviral treatment in HIV patients in Ethiopia. Epidemiology and infection 2015, 143(5):1048-1058.

6. Sebastião E, Chodzko-Zajko W, Schwingel A: An In-Depth Examination of Perceptions of Physical Activity in Regularly Active and Insufficiently Active Older African American Women: A Participatory Approach. PloS one 2015, 10(11):e0142703.

7. Joseph RP, Ainsworth BE, Keller C, Dodgson JE: Barriers to Physical Activity Among African American Women: An Integrative Review of the Literature. Women \& health 2015, 55(6):679-699.

8. John B, Todd J, Mboya I, Mosha M, Urassa M, Mtuy T: Physical activity and associated factors from a cross-sectional survey among adults in northern Tanzania. BMC public health 2017, 17(1):588.

9. Moran M, Van Cauwenberg J, Hercky-Linnewiel R, Cerin E, Deforche B, Plaut P: Understanding the relationships between the physical environment and physical activity in older adults: a systematic review of qualitative studies. The international journal of behavioral nutrition and physical activity 2014, 11:79.

10. Whitt-Glover MC, Keith NR, Ceaser TG, Virgil K, Ledford L, Hasson RE: A systematic review of physical activity interventions among African American adults: evidence from 2009 to 2013. Obes Rev 2014, 15 Suppl 4:125-145.

11. Montgomery CA, Henning KJ, Kantarzhi SR, Kideckel TB, Yang CF, O'Brien KK: Experiences participating in a community-based exercise programme from the perspective of people living with HIV: a qualitative study. BMJ open 2017, 7(4):e015861.

12. Gomes Neto M, Conceicao CS, Oliveira Carvalho V, Brites C: Effects of Combined Aerobic and Resistance Exercise on Exercise Capacity, Muscle Strength and Quality of Life in HIV-Infected 
Patients: A Systematic Review and Meta-Analysis. PloS one 2015, 10(9):e0138066.

13. Ley C, Barrio MR: A narrative review of research on the effects of physical activity on people living with HIV and opportunities for health promotion in disadvantaged settings. African journal of AIDS research: AJAR 2012, 11(2):123-133.

14. Marcus BH, Simkin LR: The transtheoretical model: applications to exercise behavior. Medicine and science in sports and exercise 1994, 26(11):1400-1404.

15. Hashemzadeh M, Rahimi A, Zare-Farashbandi F, Alavi-Naeini AM, Daei A: Transtheoretical Model of Health Behavioral Change: A Systematic Review. Iranian journal of nursing and midwifery research 2019, 24(2):83-90.

16. Rosenstock IM, Strecher VJ, Becker MH: Social learning theory and the Health Belief Model. Health education quarterly 1988, 15(2):175-183.

17. Hutchison AJ, Breckon JD, Johnston LH: Physical activity behavior change interventions based on the transtheoretical model: a systematic review. Health education \& behavior: the official publication of the Society for Public Health Education 2009, 36(5):829-845.

18. McLeroy KR, Bibeau D, Steckler A, Glanz K: An ecological perspective on health promotion programs. Health education quarterly 1988, 15(4):351-377.

19. Understanding qualitative research in health care. Drug and therapeutics bulletin 2017, 55(2):21-24.

20. Sallis JF, Cervero RB, Ascher W, Henderson KA, Kraft MK, Kerr J: An ecological approach to creating active living communities. Annual review of public health 2006, 27:297-322.

21. Ley C, Barrio MR, Leach L: Social-Ecological, Motivational and Volitional Factors for Initiating and Maintaining Physical Activity in the Context of HIV. The open AIDS journal 2015, 9:96-103.

22. Jeremiah K, Filteau S, Faurholt-Jepsen D, Kitilya B, Kavishe BB, Krogh-Madsen R, Olsen MF, Changalucha J, Rehman AM, Range $\mathrm{N}$ et al: Diabetes prevalence by HbA1c and oral glucose tolerance test among HIV-infected and uninfected Tanzanian adults. PloS one 2020, 15(4):e0230723.

23. Ministry of Finance NbosaMC: Mwanza City Socio-economic profile. In. Edited by Office MCPaRc, 2016 edn. Mwanza: Mwanza City council; 2016: 179.

24. Fitzgerald N, Spaccarotella K: Barriers to a Healthy Lifestyle: From Individuals to Public Policy - An Ecological Perspective. Journal of Extension 2009, 47:1FEA3.

25. Britten N: Qualitative interviews in medical research. BMJ 1995, 311(6999):251-253.

26. Golden SD, Earp JA: Social ecological approaches to individuals and their contexts: twenty years of health education \& behavior health promotion interventions. Health education \& behavior: the official publication of the Society for Public Health Education 2012, 39(3):364-372.

27. Elo S, Kyngäs H: The qualitative content analysis process. J Adv Nurs 2008, 62(1):107-115.

28. Piercy KL, Bevington F, Vaux-Bjerke A, Hilfiker SW, Arayasirikul S, Barnett EY: Understanding Contemplators' Knowledge and Awareness of the Physical Activity Guidelines. Journal of physical activity \& health 2020, 17(4):404-411. 
29. Cameron C, Craig CL, Bull FC, Bauman A: Canada's physical activity guides: has their release had an impact? Canadian journal of public health = Revue canadienne de sante publique 2007, 98 Suppl 2:S161-169.

30. Wong MK, Cheng SYR, Chu TK, Lee CN, Liang J: Hong Kong Chinese adults' knowledge of exercise recommendations and attitudes towards exercise. BJGP open 2017, 1(2):bjgpopen17X100929.

31. Spencer RA, Rehman L, Kirk SF: Understanding gender norms, nutrition, and physical activity in adolescent girls: a scoping review. The international journal of behavioral nutrition and physical activity 2015, 12:6.

32. Richman EL, Shaffer DR: IF YOU LET ME PLAY SPORTS: How Might Sport Participation Influence the Self-Esteem of Adolescent Females? Psychology of Women Quarterly 2000, 24(2):189-199.

33. Yiga P, Seghers J, Ogwok P, Matthys C: Determinants of dietary and physical activity behaviours among women of reproductive age in urban sub-Saharan Africa: a systematic review. The British journal of nutrition 2020, 124(8):761-772.

34. Cheng LA, Mendonça G, Farias Júnior JCd: Physical activity in adolescents: analysis of the social influence of parents and friends. Jornal de Pediatria 2014, 90(1):35-41.

35. Larsen BA, Strong D, Linke SE: The association between family and friend integration and physical activity: results from the NHIS. International journal of behavioral medicine 2014, 21(3):529-536.

36. Mendonça G, Cheng LA, Mélo EN, de Farias Júnior JC: Physical activity and social support in adolescents: a systematic review. Health Education Research 2014, 29(5):822-839.

37. Paes Lda S, Borges JP, Dos Santos FM, de Oliveira TP, Dupin JG, Harris EA, Farinatti P: Effects of a 2Year Supervised Exercise Program Upon the Body Composition and Muscular Performance of HIVInfected Patients. The open AIDS journal 2015, 9:80-88.

38. Mathew Joseph N, Ramaswamy P, Wang J: Cultural factors associated with physical activity among U.S. adults: An integrative review. Applied nursing research: ANR 2018, 42:98-110.

39. Wright CH, Longenecker CT, Nazzindah R, Kityo C, Najjuuko T, Taylor K, Rentrope CR, Webel A: A Mixed Methods, Observational Investigation of Physical Activity, Exercise, and Diet Among Older Ugandans Living With and Without Chronic HIV Infection. The Journal of the Association of Nurses in AIDS Care: JANAC 2020.

40. Johs NA, Kellar-Guenther Y, Jankowski CM, Neff H, Erlandson KM: A qualitative focus group study of perceived barriers and benefits to exercise by self-described exercise status among older adults living with HIV. BMJ open 2019, 9(3):e026294.

41. Li A, McCabe T, Silverstein E, Dragan S, Salbach NM, Zobeiry M, Beldick S, Godi C, O'Brien KK: Community-Based Exercise in the Context of HIV: Factors to Consider when Developing and Implementing Community-Based Exercise Programs for People Living with HIV. Journal of the International Association of Providers of AIDS Care (JIAPAC) 2017, 16(3):267-275.

42. welfare Mohas: Tanzania national guidelines for management of HIV and AIDS. In. Edited by program NAc, Third edition edn. Tanzania: Ministry of Health and social Welfare; 2009: 328. 
43. Johansson KA, Jerene D, Norheim OF: National HIV treatment guidelines in Tanzania and Ethiopia: are they legitimate rationing tools? Journal of medical ethics 2008, 34(6):478-483.

44. Ministry of Health CD, Gender, Elderely and Children: National guidelines for Managemnt of HIV and AIDS. In. Edited by Programme NAc, Six Edition edn. Tanzania: Ministry of Health, Community Development, Gender, Elderely and Children; 2017: 344.

45. Dang AK, Nguyen LH, Nguyen AQ, Tran BX, Tran TT, Latkin CA, Zhang MWB, Ho RCM: Physical activity among HIV-positive patients receiving antiretroviral therapy in Hanoi and Nam Dinh, Vietnam: a cross-sectional study. BMJ open 2018, 8(5):e020688.

\section{Figures}

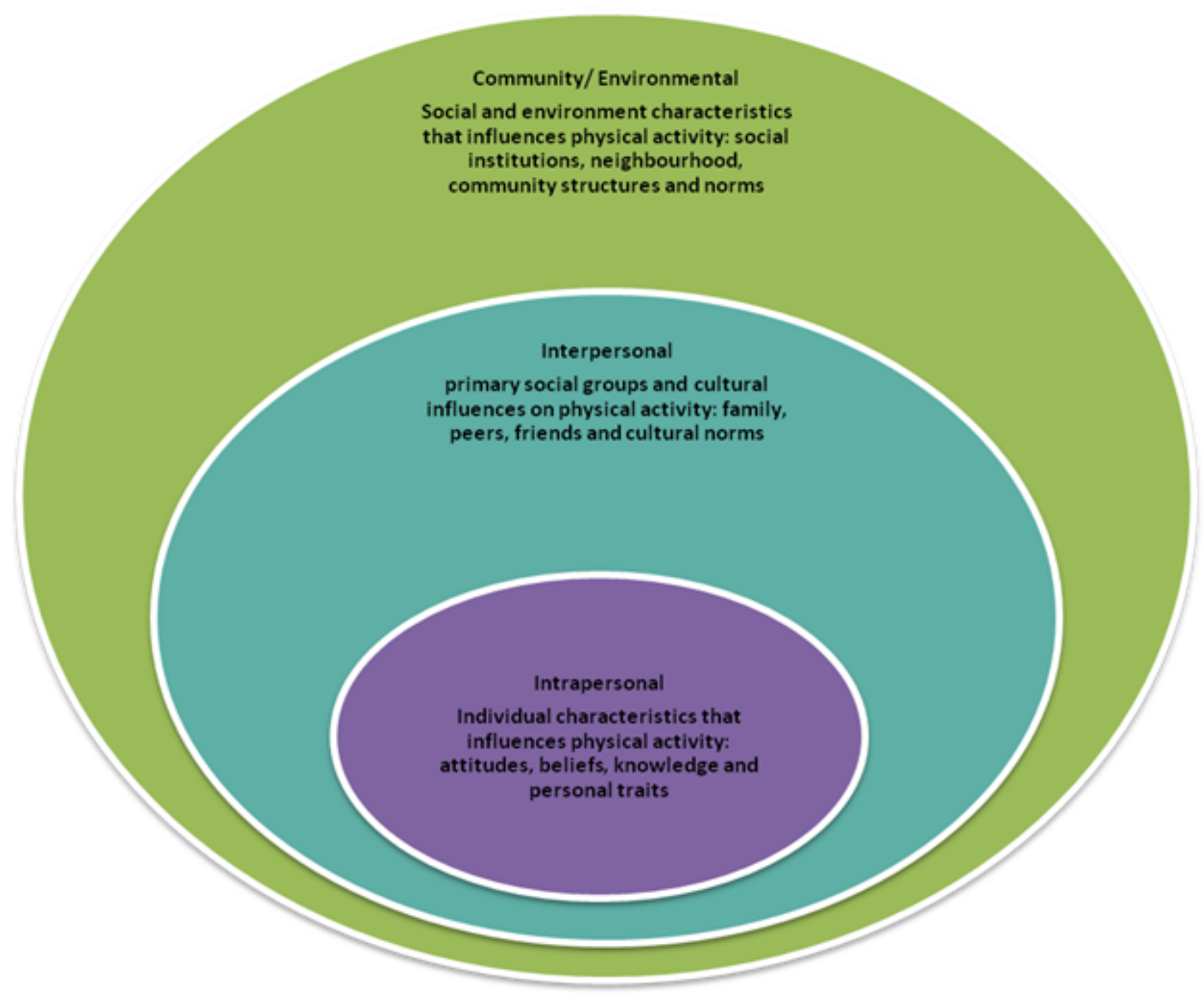

Figure 1

Social-ecological model used to describe perceptions, facilitators and barriers of physical activity [18] 